\title{
Under-Determined Non-cartesian MR Reconstruction with Non-convex Sparsity Promoting Analysis Prior
}

\author{
Angshul Majumdar and Rabab K. Ward \\ Department of Electrical and Computer Engineering, University of British Columbia \\ \{angshulm, rababw\} @ece.ubc.ca
}

\begin{abstract}
This work explores the problem of solving the MR reconstruction problem when the number of K-space samples acquired in a non-Cartesian grid is considerably less than the resolution (number of pixels) of the image. Mathematically this leads to the solution of an under-determined and ill-posed inverse problem. The inverse problem can only be solved when certain additional/prior assumption is made about the solution. In this case, the prior is the sparsity of the MR image in the wavelet domain. The non-convex lp-norm ( ) of the wavelet coefficient is a suitable metric for sparsity. Such a prior can appear in two forms - in the synthesis prior formulation, the wavelet coefficients of the image is solved for while in the analysis prior formulation the actual image is solved for. Traditionally the synthesis prior formulation is more popular. However, in this work we will show that the analysis prior formulation on redundant wavelet transform provides better MR reconstruction results compared to the synthesis prior formulation.
\end{abstract}

Keywords: MRI, image reconstruction, non-convex optimization.

\section{Introduction}

In Magnetic Resonance Imaging (MRI) the K-space (Fourier frequency) samples are collected. The problem is to reconstruct the image from the K-space samples. The reconstruction is trivial (inverse Discrete Fourier Transform) when the K-space is densely sampled. But such dense sampling is time consuming. In this work, we investigate the possibility to reduce MR acquisition times by reducing the number of $\mathrm{K}$ space samples.

We are particularly interested in non-Cartesian K-space sampling like the radial, spiral or rosetta. For such sampling, the relationship between the image space and the $\mathrm{K}$-space is given by [1],

$$
y_{m \times 1}=F_{m \times n} x_{n \times 1}+\eta_{n \times 1}, m<n
$$

where, $\mathrm{y}$ is the collected K-space samples, $\mathrm{F}$ is the Non Uniform Fast Fourier Transform (NUFFT), $\mathrm{x}$ is the image and $\eta$ is the noise, assumed to be Gaussian with mean zero.

The inverse problem (1) is under-determined, and does not have a unique solution. However, if additional information is available regarding the character solution, one 
may be able to pick up a unique solution satisfying that character. In [2] it is argued that the MR image is sparse in Wavelet domain. Therefore, a solution to (1) was seek which had a sparse representation in the wavelet domain. It was shown experimentally in [2] that the said assumption is valid and leads to impressive image reconstruction results from under-sampled K-space.

Incorporating the wavelet transform into the inverse problem (1) leads to the following equation,

$$
y=F W^{T} \alpha+\eta
$$

Where $\mathrm{W}$ is the forward wavelet transform $\left(\mathrm{W}^{\mathrm{T}}\right.$ is the inverse) and $\alpha$ is the transform coefficients (assumed to be sparse for MR images).

Assume that the vector $\alpha$ is $\mathrm{k}$-sparse (i.e. k non-zeroes and n-k zeroes). Ideally one would solve the following optimization problem in order to search for the sparsest solution.

$$
\min \|\alpha\|_{0} \text { subject to }\left\|y-F W^{T} \alpha\right\|_{2} \leq \sigma
$$

where $\|.\|_{0}$ is the number of non-zeroes in the vector and $\sigma$ is the standard deviation of noise.

Since the wavelet coefficient of the MR image is assumed to be k-sparse (i.e. $\mathrm{k}$ non-zeroes and $\mathrm{n}-\mathrm{k}$ zeroes), only $m \approx 2 k$ samples are needed for obtaining the solution of (7) via (8). Unfortunately (8) is an NP hard problem. But theoretical study [3] proves that - (i) if a linear system has a sparse solution, the solution is typically unique; and (ii) the solution can be obtained by the following convex optimization (4) instead of an NP hard problem.

$$
\min \|\alpha\|_{1} \text { subject to }\left\|y-F W^{T} \alpha\right\|_{2} \leq \sigma
$$

This is a very strong result which guarantees recovery of the image by a tractable algorithm, but on the other hand, the number of samples required is larger $m \approx C k \log (n / k)$. In [2], the MR reconstruction was based on (4).

Our aim is to reduce the MR acquisition time as much as possible, which in turn is proportional to the number of K-space samples. Therefore to reduce MR acquisition time, we should be able to reconstruct the images with as few samples as possible. Between the two extremes (3) (very few samples but NP hard reconstruction algorithm) and (4) (considerably larger number of samples but easy reconstruction algorithm) there exists reconstruction algorithms based on non-convex optimization.

$$
\min \|\alpha\|_{p} \text { subject to }\left\|y-F W^{T} \alpha\right\|_{2} \leq \sigma, 0<p<1
$$

Such non-convex methods require samples that are intermediate between the requirements of (3) and (4), $m \approx C_{1} k+p C_{2} k \log (n / k)$ [4]. As the value of 'p' is reduced, the number of samples required for reconstruction reduces smoothly.

Algorithms for solving non-convex optimization problems (5) are not more complex than solving convex optimization problems, but are not guaranteed to reach a global mimima. However, (5) is not any non-convex problem, rather it is quasiconvex. Convergence proofs for quasi-convex formulations can be found in [5]. 
The formulation discussed so far (3-5) is called the synthesis prior formulation. In this formulation, the sparse wavelet coefficients of the MR image are solved for. Once the wavelet domain representation is obtained, the image is reconstructed via the wavelet synthesis equation $(x=W \alpha)$. The synthesis prior formulation (for the convex case, i.e. $\mathrm{p}=1$ ) has been previously used [2] for MR image reconstruction. However, a recent work [6] has pointed out that an alternate analysis prior (for the convex case, $\mathrm{p}=1$ ) formulation (6) yields better reconstruction results when redundant wavelets are used instead of orthogonal wavelets (for the orthogonal case, the two formulations are the same). The experiments in [6] were carried out on synthetically generated data.

$$
\min \|W x\|_{p} \text { subject to }\|y-F x\|_{2} \leq \sigma, 0<p<1
$$

The analysis prior formulation solves for the image directly rather than the wavelet coefficients. In this work, we show that the non-convex analysis prior when employed on redundant wavelets yield better MR reconstruction results compared to synthesis prior on orthogonal wavelets.

There are mane algorithms to solve the convex optimization problem with synthesis prior (4). There only a single general purpose algorithm [7,8] to solve the nonconvex synthesis prior problem (5). This algorithm is called the Iterative Reweighted Least Squares (IRLS) algorithm. However, IRLS is a second order method and is consequently very slow. To overcome the limitations of speed we propose a new algorithm for solving the non-convex synthesis prior problem.

There are a few algorithms for solving the convex optimization problem for analysis priors, but none for the non-convex version. Therefore, we had to develop a new algorithm to solve it. Our proposed algorithms for non-convex optimization on analysis/synthesis priors are based on the Majorization-Minimization approach.

The rest of the paper is organized into several sections. The next section contains informal derivations of the non-convex algorithms proposed in this paper. The experimental results are presented in Section 3. Finally in Section 4, the conclusions of this work are discussed.

\section{Optimization Algorithms}

The constrained problems (5) and (6) are hard to solve. Rather, we proceed to solve their unconstrained versions,

$$
\begin{gathered}
\min \left\|y-F W^{T} \alpha\right\|_{2}^{2}+\lambda\|\alpha\|_{p}^{p} \\
\min \|y-F x\|_{2}^{2}+\lambda\|W x\|_{p}^{p}
\end{gathered}
$$

The parameter $\lambda$ in the equations (7) and (8) are related to $\sigma$ in (5) and (6), but the relationship is not analytical. For the time being we concentrate on the solution of equations (7) and (8). How to make the solutions of (7) and (8) reach those of (5) and (6) iteratively, will be discussed later. 


\subsection{Majorization-Minimization}

The Majorization-Minimization framework is outlined in [9]. The problems (7) and (8) do not have a closed form solution and has to be solved iteratively. In the Majorization-Minimization (MM) approach, at each iteration the function to be minimized is replaced by a surrogate function which has a higher functional value at all points except at its minima; the value of the surrogate function and the original function are the same at the minima. The surrogate function is easier to solve than the original function. The idea behind this approach is to construct such a surrogate function so that its solution after each iteration, is closer to the desired solution.

\section{$\underline{\text { MM Framework }}$}

Let $\mathrm{J}(\mathrm{x})$ be the original function that is to be minimized.

Initialize: iteration counter $\mathrm{k}=0$; initial estimate $\mathrm{x}_{0}$.

Repeat the following steps until a suitable exit criterion is met.

1. Chose $G_{k}(x)$ such that:

\section{1. $G_{k}(x) \geq J(x), \forall x$}

1.2. $G_{k}\left(x_{k}\right)=J\left(x_{k}\right)$

2. Set: $x_{k+1}=\min G_{k}(x)$

Set: $\mathrm{k}=\mathrm{k}+1$ and return to step 1 .

\subsection{Landweber Iterations}

Let us consider the minimization of the following optimization problem,

$$
J(x)=\|y-M x\|_{2}^{2}
$$

Here $\mathrm{M}$ is a generic matrix, for (7) it needs to be replaced by $F W^{T}$ and for (8) it needs to be replaced by $F$.

For this minimization, the surrogate function $G_{k}(x)$ is chosen to be,

$$
G_{k}(x)=\|y-M x\|_{2}^{2}+\left(x-x_{k}\right)^{T}\left(a I-M^{T} M\right)\left(x-x_{k}\right)
$$

To maintain strict convexity $a>\max e i g\left(M^{T} M\right)$.

$G_{k}(x)$ is minimized by Landweber iterations,

$$
x_{k+1}=x_{k}+\frac{1}{a} M^{T}\left(y-M x_{k}\right)
$$

Using this update formula, $G_{k}(x)$ can be expressed as,

$$
G_{k}(x)=a\left\|x-x_{k+1}\right\|_{2}^{2}-a x_{k+1}^{T} x_{k+1}+y^{T} y+x_{k}^{T}\left(a I-M^{T} M\right) x_{k}
$$


Note that all the terms apart from the first term are independent of $x$ and does not play any part in the minimization.

\subsection{Synthesis Prior Algorithm}

The problem to be solved is,

$$
J(x)=\left\|y-F W^{T} \alpha\right\|_{2}^{2}+\lambda\|\alpha\|_{p}^{p}
$$

After the discussion in the previous sub-section the choice of $G_{k}(x)$ is quite obvious,

$$
G_{k}(x)=\left\|\alpha-\alpha_{k+1}\right\|_{2}^{2}+\frac{\lambda}{a}\|\alpha\|_{p}^{p}
$$

where $\alpha_{k+1}=\alpha_{k}+\frac{1}{a} W F^{T}\left(y-F W^{T} \alpha_{k}\right)$

The minimizer of (16) is the following (we omit the derivation owing to limitations in space),

$$
\alpha=\operatorname{signum}\left(\alpha_{k+1}\right) \max \left(0,\left|\alpha_{k+1}\right|-\frac{\lambda}{2 a} \operatorname{p.Diag}\left(\left|\alpha_{k+1}\right|^{p-2}\right)\right)
$$

\subsection{Analysis Prior Algorithm}

We are interested in solving the following problem,

$$
J(x)=\|y-F x\|_{2}^{2}+\lambda\|W x\|_{p}^{p}
$$

Following the discussion in sub-section 3.2 the choice of $G_{k}(x)$ is,

$$
\begin{aligned}
& G_{k}(x)=\left\|x-x_{k+1}\right\|_{2}^{2}+\frac{\lambda}{a}\|W x\|_{p}^{p} \\
& \text { where } x_{k+1}=x_{k}+\frac{1}{a} F^{T}\left(y-F x_{k}\right)
\end{aligned}
$$

Owing to limitations in space, we skip the derivations. (17 is minimized by the following updates,

$$
\begin{aligned}
& z_{k+1}=\left(\frac{2 a}{\lambda} D^{-1}+c I\right)^{-1}\left(c z_{k}+H\left(x_{k}-H^{T} z_{k}\right)\right), \text { where } c>\max \operatorname{eig}\left(W^{T} W\right) \\
& x_{k+1}=x_{k}-H^{T} z_{k+1} \\
& x_{k}=x_{k-1}+\frac{1}{a} A^{T}\left(y-A x_{k-1}\right)
\end{aligned}
$$

\subsection{Constrained Optimization via Cooling}

Theoretically the constrained forms (5) and (6) and the unconstrained forms (7) and (8) are equivalent for correct choice of $\lambda$ and $\sigma$. However, for all practical case, it is 
not possible to find the relation between the two explicitly. Owing to the smoothness of the Pareto curve, solving the unconstrained problems for a decreasing sequence of $\lambda$ is guaranteed to reach the solution desired by the constrained form [10]. Based on this idea, we use a cooling algorithm to solve (5) and (6) via iteratively solving (7) and (8).

1. Choose a high value of $\lambda$ initially.

2. Solve the unconstrained optimization (7)/(8) for the given value of $\lambda$.

3. Decrease the value of $\lambda$ and go back to step 1 .

4. Continue steps 2 and 3, until the mismatch ( $\left.\left\|y-F W^{T} \alpha\right\|_{2} /\|y-F x\|_{2}\right)$ between the data and the solution is less than $\sigma$.

\section{Experimental Evaluation}

The experimental evaluation was carried out on two real brain slices - Brainweb and $\mathrm{NIH}$. The data is assumed to be noiseless. The experiments were simulated for radial scan lines in the k-space. The number of samples in k-space was always less than the number of pixels in the reconstructed image. As mentioned earlier the mapping from the Cartesian image space to the non-Cartesian k-space is the NUFFT [1]. Haar wavelets are used as the sparsifying basis. The normalized mean squared error between the ground-truth and the reconstructed image is used as an evaluation metric.

For all experiments it was found that the best reconstruction results were obtained at $\mathrm{p}=0.8$ for both the analysis and the synthesis prior problems. In the following tables the reconstruction results for one slice each from Brainweb (Table 1) and NIH (Table 2) are reported for different sampling ratios (ratio of number of samples in K-space to the number of pixels in image).

Table 1. Reconstruction Results for Brainweb Slice

\begin{tabular}{|l|l|l|l|l|l|l|}
\hline \multirow{2}{*}{ Algorithm } & \multicolumn{6}{|l|}{ Sampling Ratio (in percent) } \\
\cline { 2 - 7 } & 19.94 & 23.83 & 27.73 & 31.62 & 35.52 & 39.41 \\
\hline $\begin{array}{l}\text { Orthogonal } \\
\text { Synthesis }\end{array}$ & 0.155 & 0.132 & 0.114 & 0.099 & 0.092 & 0.088 \\
\hline $\begin{array}{l}\text { Redundant } \\
\text { Synthesis }\end{array}$ & 0.331 & 0.227 & 0.197 & 0.278 & 0.247 & 0.207 \\
\hline $\begin{array}{l}\text { Redundant } \\
\text { Analysis }\end{array}$ & $\mathbf{0 . 1 0 5}$ & $\mathbf{0 . 0 9 1}$ & $\mathbf{0 . 0 7 9}$ & $\mathbf{0 . 0 7 1}$ & $\mathbf{0 . 0 6 8}$ & $\mathbf{0 . 0 6 0}$ \\
\hline
\end{tabular}

Table 2. Reconstruction Results for NIH Slice

\begin{tabular}{|l|l|l|l|l|l|l|}
\hline \multirow{2}{*}{ Algorithm } & \multicolumn{6}{|l|}{ Sampling Ratio (in percent) } \\
\cline { 2 - 7 } & 19.94 & 23.83 & 27.73 & 31.62 & 35.52 & 39.41 \\
\hline $\begin{array}{l}\text { Orthogonal } \\
\text { Synthesis }\end{array}$ & 0.258 & 0.233 & 0.212 & 0.196 & 0.183 & 0.170 \\
\hline $\begin{array}{l}\text { Redundant } \\
\text { Synthesis }\end{array}$ & 0.484 & 0.385 & 0.376 & 0.376 & 0.343 & 0.303 \\
\hline $\begin{array}{l}\text { Redundant } \\
\text { Analysis }\end{array}$ & $\mathbf{0 . 2 0 3}$ & $\mathbf{0 . 1 8 4}$ & $\mathbf{0 . 1 6 7}$ & $\mathbf{0 . 1 5 6}$ & $\mathbf{0 . 1 4 4}$ & $\mathbf{0 . 1 3 4}$ \\
\hline
\end{tabular}


Tables 1-2 confirm that minimizing the $l_{p}$-norm on a redundant analysis prior gives the best MR reconstruction results. We also provide a qualitative comparison of the reconstruction results; owing to the limitations in space we provide results only for the NIH slice (since it is more challenging).

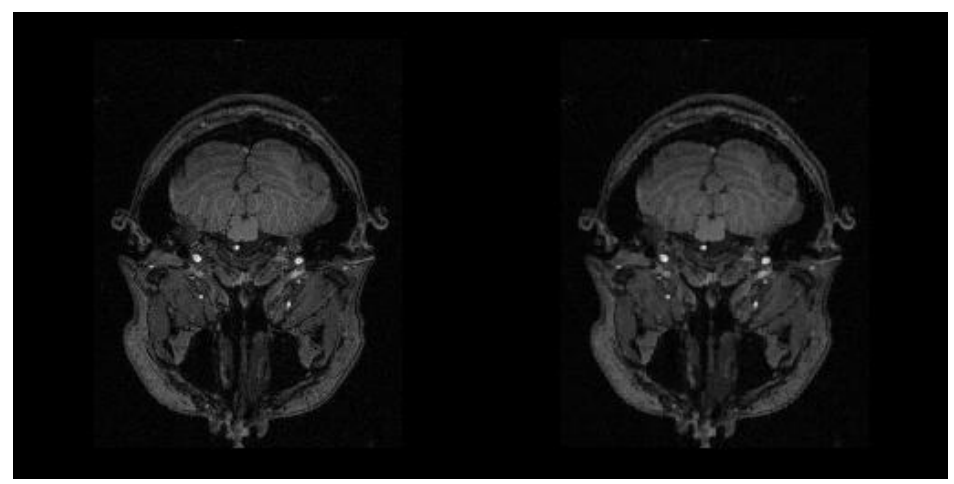

(a)

(b)

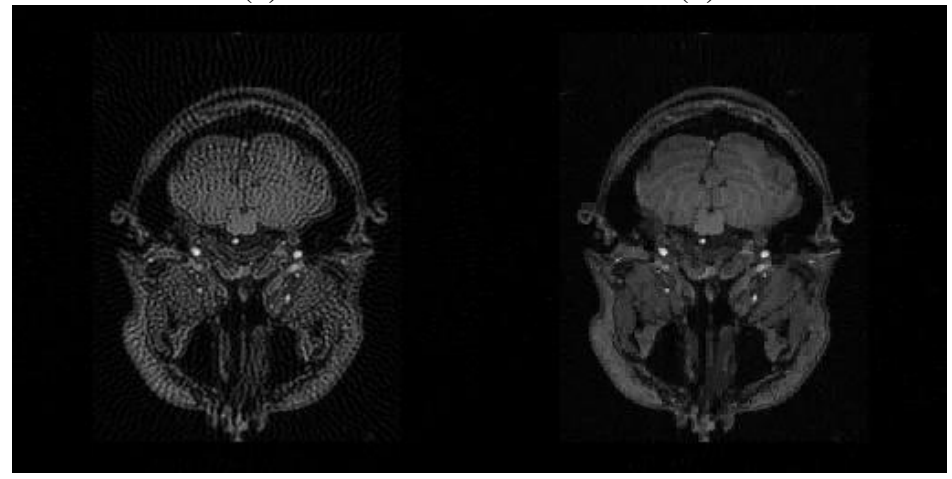

(c)

(d)

Fig. 1. NIH slice - (a) Ground truth; (b) Orthogonal Haar Synthesis; (c) Redundant Haar Synthesis and (d) Redundant Haar Analysis.

On close inspection the reconstructed images show blocky artifacts. This is because, we are using Haar wavelets for reconstruction. More sophisticated wavelets like Dualtree or Fractional Spline will reduce such artifacts.

\section{Conclusion}

In this work we look at the problem of reducing the MR acquisition times from a signal processing perspective. The acquisition time for the scanners are directly proportional to the number of number of samples collected in the K-space. Therefore in order to reduce the acquisition time the number of samples needs to be reduced. However, this leads to under-determinacy of the reconstruction problem. Inspite of having an under-determined system of linear equations it is possible to exploit the sparsity of 
the MR images in the wavelet domain to obtain a unique solution via a non-convex optimization problem.

The optimization problem can be formulated in two flavours - sparsity promoting analysis prior and sparsity promoting synthesis prior. In this work, we show that better reconstruction results can be obtained with analysis prior algorithm using redundant (Haar) wavelets.

Non-convex algorithms for sparsity promoting priors is not a well developed subject. There is only a single algorithm [7, 8] to solve the synthesis prior problem, which is computationally slow; hence we did not employ it. In this paper we propose a new fast algorithm for solving the non-convex synthesis prior problem. The analysis prior problem in the non-convex setting was never encountered earlier, therefore we developed an efficient first order algorithm to solve this problem.

\section{References}

[1] Fessler, J.A.: On NUFFT-based gridding for non-Cartesian MRI. Jour. Magn. Reson. 188(2), 191-195 (2007)

[2] Lustig, M., Donoho, D.L., Pauly, J.M.: Sparse MRI: The Application of Compressed Sensing for Rapid MR Imaging. Magnetic Resonance in Medicine 58(6), 1182-1195 (2007)

[3] Donoho, D.L.: For most large underdetermined systems of linear equations the minimal $1_{1}$-norm solution is also the sparsest solution. Comm. on Pure and Applied Maths 59(6), 797-829 (2006)

[4] Chartrand, R.: Nonconvex compressed sensing and error correction. In: IEEE Int. Conf. on Acoustics, Speech, and Signal Processing (ICASSP), Honolulu, Hawaii (April 2007)

[5] Luenberger, D.G.: Quasi-convex programming. SIAM Journal on Applied Mathematics 16, 1090 (1968)

[6] Selesnick, W., Figueiredo, M.A.T.: Signal restoration with overcomplete wavelet transforms: comparison of analysis and synthesis priors. In: Proceedings of SPIE, vol. 7446, Wavelets XIII (2009)

[7] Majumdar, A., Ward, R.K.: Non-Convex Compressed Sensing from Noisy Measurements. The Open Signal Processing Journal 2, 40-45 (2009)

[8] Chartrand, R., Yin, W.: Iteratively reweighted algorithms for compressive sensing. In: ICASSP pp. 3869-3872 (2008)

[9] http://cnx.org/content/m32168/latest/

[10] Hennenfent, G., van den Berg, E., Friedlander, M.P., Herrmann, F.J.: New insights into one-norm solvers from the pareto curve. Geophysics 73(4) (2008) 